\title{
AVALIAÇÃO DA RESISTÊNCIA A FADIGA DOS ENGINEERED CEMENTITIOUS COMPOSITES (ECC), REFORÇADOS COM FIBRA DE POLIPROPILENO E PRODUZIDOS COM ADIÇÃO DE CINZA DE CASCA DE ARROZ.
}

\author{
Alceu Lopes de Freitas Júnior ${ }^{1 *}$, Mônica Regina Garcez ${ }^{2}$ \\ 1 Programa de Pós-Graduação em Ciência e Engenharia de Materiais, Universidade Federal de Pelotas, 96010-000, Brasil. \\ 2 Núcleo de Estudos em Materiais Compósitos Avançados, Universidade Federal de Pelotas, 96010-000, Brasil.
}

*E-mail: alceu.junior@ufpel.edu.br

\section{RESUMO}

O Engineered Cementitious Composites - ECC - é um tipo especial de compósito cimentício de alto desempenho reforçado com fibra, cuja principal característica é a alta ductilidade. O traço de ECC é composto por cimento, material pozolânico, agregado miúdo e fibras. Este estudo avaliou a resistência à fadiga de traços de Engineered Cementitious Composites reforçados com fibras de polipropileno - PPECC - e cuja composição da matriz cimentícia teve a incorporação de cinza de casca de arroz como material suplementar. Para isto, foram produzidos corpos de prova com substrato de concreto para pavimento e overlay com o traço de PPECC desenvolvido. Os corpos de prova foram submetidos aos ensaios de flexão a quatro pontos (estático e cíclico). Compararam-se os resultados obtidos no ensaio estático dos traços de PPECC com os resultados obtidos neste mesmo ensaio com corpos de prova produzidos somente por concreto para pavimento. O PPECC deste estudo apresentou desempenho satisfatório aos ensaios de flexão estática. Foram analisados os resultados dos ensaios cíclicos em diferentes intervalos de tensões. Com os resultados variação da tensão x números de ciclos, obtidos no ensaio cíclico, foi avaliado o comportamento à fadiga do compósito. Nos ensaios de flexão cíclica, pode-se observar que quanto menor o nível de tensão aplicada, menor é o número de fissuras desenvolvidas no overlay de PPECC e este não desenvolve suas potencialidades. Como conclusão, temos que o $E C C$ constitui-se em um compósito alternativo com viabilidade de aplicação em overlays sobre base de CP sujeitos a esforços cíclicos.

Palavras-chave: Compósitos cimentícios. Compósitos cimentícios com adição de fibras de polipropileno. Fadiga em compósito cimentício.

\section{Introdução}

O Engineered Cementitious Composites - ECC, também chamado de compósito cimentício flexível, é uma classe de compósito cimentício de alto desempenho reforçado com fibras, cuja principal característica é a altíssima ductilidade. As matérias primas constituintes são: cimento, areia, fibra e plastificantes.

Para produção destes compósitos de elevada capacidade de deformação faz-se necessário o emprego de maiores quantidades de cimento, de duas a três vezes mais que nos concretos convencionais. A fim de reduzir a quantidade de cimento nas misturas $\mathrm{e}$ proporcionar melhorias no comportamento do material, são incorporados materiais pozolânicos suplementares na matriz cimentícia.

Este compósito cimentício consiste num material muito deformável com um volume moderado de fibras (da ordem de $2 \%$ em relação ao volume total), sendo possível explorar a ductibilidade na resposta final de uma estrutura. Os ECC podem atingir deformações da ordem de 5\%, 500 vezes maiores que um concreto convencional, atingindo deflexões elevadas em ensaios de flexão a quatro pontos [1].
A teoria de concepção é baseada na micromecânica relacionada a propriedades macroscópicas do compósito, e as propriedades individuais de cada fase dos mesmos, ou seja, da fibra, da matriz e da interface fibra-matriz. As vantagens da ductibilidade no estado de dureza e da flexibilidade no estado fresco fazem do ECC um material atrativo para largas escalas de aplicação.

A alta capacidade de deformação após atingir a tensão máxima de carregamento gera o comportamento chamado de strain-hardening, característico por transferir o carregamento entre as fibras gerando muitas fissuras de pequenas aberturas. Essas múltiplas microfissuras acontecem antes da ruptura ou arrancamento das fibras. Sendo assim, no momento que a carga é transferida para a fibra que conduz a carga à matriz, surgem pequenas fissuras em diferentes pontos; a repetição dessa ação gera deformações superiores, resistência elevada e o material tende há resistir mais tempo antes de romper [2]. Assim, o comportamento strain-hardening é o responsável pela característica mais importante deste compósito, pois o mesmo garante a alta ductilidade, permitindo que o compósito sofra elevadas deformações sem romper [3]. 
Devido a estas características, o ECC pode ser utilizado como camada de reforço, de forma eficiente, para pavimentos de concreto deteriorados, quando submetidos a esforços cíclicos, os quais geram a fadiga do material [4]. O estudo do comportamento mecânico dos materiais sob fadiga permite prever até quando determinadas estruturas sujeitas a cargas dinâmicas poderão continuar sendo utilizadas sem apresentar riscos de falhas, ou ainda de que maneira pode-se retardar a propagação das trincas, visando aumentar a vida útil do material. Também visam determinar os intervalos de tempo, nos quais seriam necessárias manutenções preventivas para que algum mecanismo de falha previamente iniciado pudesse ser detectado, antes de provocar a falha total do material [5].

Neste sentido, visando uma aplicação prática do compósito como overlay para bases de concreto, uma vez que uma possibilidade de utilização do ECC é como reforço para pavimentos de concreto, e considerando a inexistência de dados relativos à aplicação de carregamento cíclico nos compósitos do tipo ECC produzidos com material disponível no Brasil, esta pesquisa verificou o comportamento à fadiga do $E C C$ reforçado com fibra de polipropileno (PP) e adição de cinza de casca de arroz (CCA) como material suplementar.

\section{Metodologia}

Foram produzidos corpos de prova compostos, com substrato de concreto tipo pavimento e overlay de ECC reforçado com fibra de polipropileno (PPECC), simulando um pavimento de concreto rígido com camada de reforço em PPECC, submetido a esforços cíclicos. Também foram produzidos corpos de prova com camada única (concreto tipo pavimento), para servir de parâmetro comparativo com corpos de prova compostos no ensaio de flexão estático.

O comportamento a fadiga do PPECC foi avaliado a partir das curvas de fadiga obtidas no ensaio de flexão a quatro pontos com carregamento cíclico. Estas curvas foram plotadas em função da diferença entre a $\sigma_{\text {máx }}-\sigma_{\text {mín, }}$ em porcentagem do módulo de ruptura do material (MOR), e número de ciclos até a ruptura, resultantes dos ensaios com os diferentes níveis de tensão aplicada. $\mathrm{O}$ valor do MOR representa a resistência à flexão das amostras submetidas ao ensaio a quatro pontos com carregamento estático. Assim, o experimento foi dividido na realização dos ensaios de flexão a quatro pontos com carregamento estático e com carregamento ciclíco.

Tabela 1 - Proporções das misturas

\subsection{Materiais}

O compósito cimentício adotado é composto por cimento (com adição de cinza de casca de arroz como material suplementar), areia, fibra de polipropileno, água e aditivo superplastificante. $\mathrm{O}$ cimento utilizado foi do tipo Portland de alta resistência inicial.

Foram empregados materiais disponíveis no mercado brasileiro: fibra de PP em substituição às fibras de PVA (utilizada nos traços originais de ECC), e CCA em substituição parcial ao cimento (material pozolânico suplementar, ao invés de cinza volante).

A cinza de casca de arroz (CCA) utilizada é oriunda do processo de queima não controlada para geração de energia, em uma indústria do Rio Grande do Sul. O traço de ECC estudado teve substituição de $30 \%$ de cimento (em volume) por esta cinza de casca de arroz, com moagem de 4 horas para redução de sua granulometria.

Para confecção dos compósitos cimentícios e do concreto tipo pavimento (CP), utilizou-se areia fina de origem natural, do tipo quartzoza. Para os pavimentos de concreto foi utilizada, como agregado graúdo, rocha basáltica britada.

$\mathrm{O}$ traço do $\mathrm{CP}$, dimensionado em função da resistência à tração na flexão, foi definido de modo a obter um valor médio de $5 \mathrm{MPa}$, concebendo o valor mínimo exigido para aplicação em pavimentos rígidos.

As proporções das misturas utilizadas no (PPECC) e no concreto para pavimento $(\mathrm{CP})$, podem ser visualizados na Tabela 1 .

\subsection{Preparação dos Corpos de Prova}

Os corpos de prova de CP, bem como, as bases dos corpos de prova compostos (camada de substrato) foram moldados com dimensões de 355x100x75 mm (Figura 1-a). Realizado o ensaio de abatimento do tronco de cone, conforme a NBR NM 67 [6], obteve-se como resultado 50 mm. Após 48h, os corpos de prova foram desmoldados (Figura 1-b), e passado o período de cura de 14 dias, aqueles destinados ao substrato foram conduzidos aos cortes. Procedeu-se um corte ao longo do eixo longitudinal, com o uso de uma serra policorte com disco diamantado (Figura 1-c), obtendo-se duas vigas de dimensões $355 \times 50 \times 75 \mathrm{~mm}$ (Figura 1-d). Na sequência, cada viga resultante do primeiro corte foi novamente cortada, desta vez no centro de

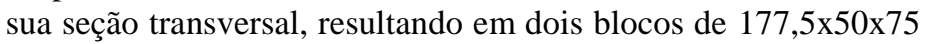
$\mathrm{mm}$. Esses dois blocos formaram o substrato para sobreposição do overlay.

\begin{tabular}{c|c|c|c|c|c|c|c|c}
\hline \multirow{2}{*}{ Denominação } & \multicolumn{9}{c}{ Quantidade de material $-\mathrm{Kg} \mathrm{m}^{-3}$} \\
\cline { 2 - 9 } & Cimento & CCA & Areia fina & Areia média & Pedra Britada & Fibra PP & Aditivo & Água \\
\hline \multirow{2}{*}{ PPECC } & 706,41 & 204,43 & 757,53 & - & - & 17,90 & 7,05 & 348,55 \\
CP & 422,00 & - & 406,80 & 610,20 & 958,40 & - & 2,80 & 164,00 \\
\hline
\end{tabular}


$\mathrm{Na}$ face resultante do corte transversal dos blocos foi colada uma fita do tipo Duct Tape, com a finalidade de assegurar uma fissura entre os blocos de uma mesma base. A seguir, os blocos foram dispostos em novas formas, com as superfícies cortadas voltadas para cima (Figura 1-e).

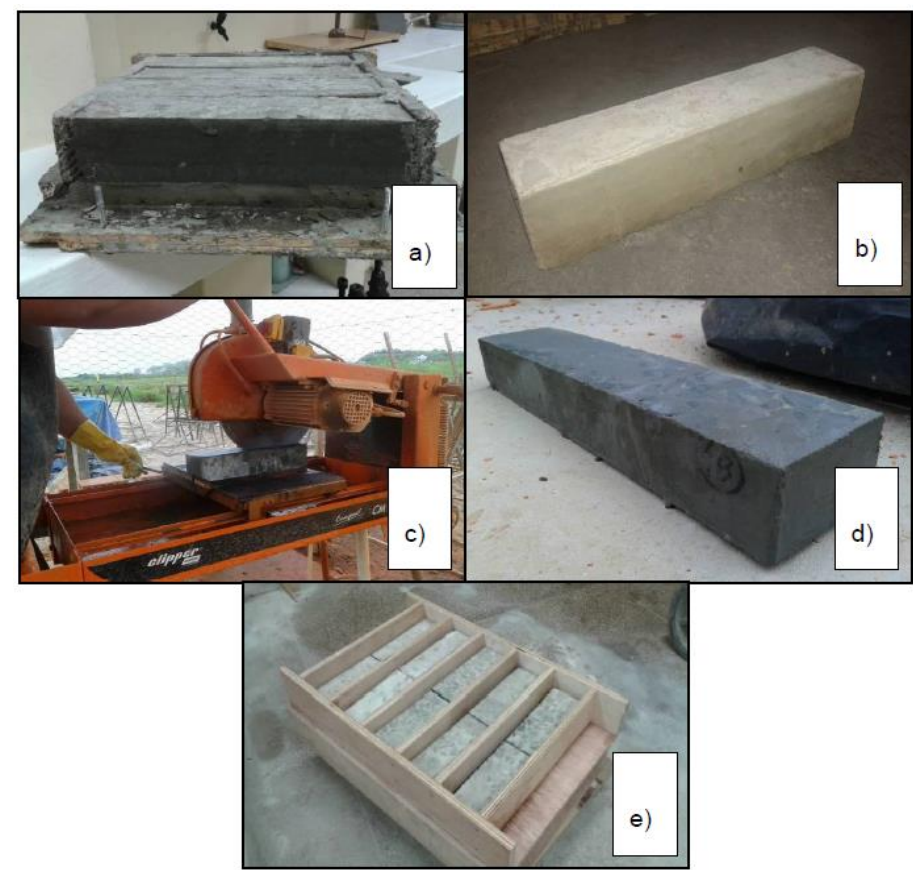

Figura 1 - (a) Corpo de prova 355x100x75mm sendo desmoldado; (b) Viga $355 \times 100 \times 75 \mathrm{~mm}$ totalmente desmoldada; (c) Corte dos corpos de prova em serra circular; (d) Viga $355 \times 50 \times 75 \mathrm{~mm}$, resultante do corte longitudinal da viga inicialmente concretada; (e) Vigas $355 \times 50 \times 75 \mathrm{~mm}$, cortadas transversalmente e dispostas em novas formas para lançamento do ECC.

Para produção do PPECC, primeiramente, foi efetuada a moagem da cinza da casca de arroz, por um período de 4 horas. Fracionados os demais materiais, foi realizada a mistura do traço do PPECC adotado em um misturador mecânico para argamassas (argamassadeira). A ordem de mistura dos materiais seguiu a seguinte sequência: materiais secos (cimento, areia e cinza da casca de arroz), água e aditivo (Figura 2-a) e, por fim, a fibra de polipropileno, adicionadas parcialmente em um intervalo de dois minutos (Figura 2-b). A argamassadeira permaneceu em velocidade de rotação baixa durante a colocação de todos os materiais. Após a colocação dos mesmos, a rotação foi alterada para a velocidade máxima, misturando os materiais por um período de mais três minutos, de modo a propiciar a dispersão total das fibras e uma mistura homogênea (Figura 2-c). Para lançamento do compósito sobre a base, as superfícies dos blocos de concreto foram limpas e umidificadas, visando garantir a aderência entre as duas camadas do corpo de prova. Nenhum tratamento superficial adicional foi executado, mantendo a rugosidade natural do concreto. A moldagem do overlay, com camada de $30 \mathrm{~mm}$, foi executada sobre o substrato de concreto, resultando nas vigas compostas, conforme apresentado na Figura 2-d.

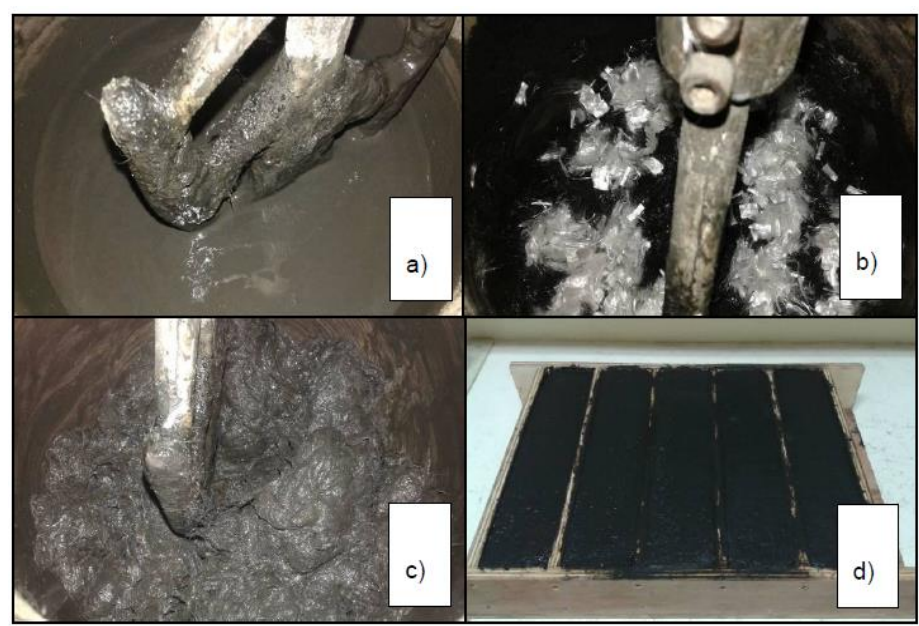

Figura 2 - (a) Mistura do cimento, areia e CCA, antes da adição da fibra; (b) Adição da fibra a mistura, de forma parcial; (c) $E C C$ com a fibra incorporada após 3 minutos de mistura; (d) Corpos de prova após a moldagem do overlay de ECC.

\subsection{Ensaio de flexão a quatro pontos}

$\mathrm{O}$ ensaio a flexão a quatro pontos seguiu as especificações da norma ASTM C78 - 02: Standard Test Method for Flexural Strength of Concrete [7].

Para o ensaio de flexão a quatro pontos com carregamento estático foram utilizados três corpos de prova de $\mathrm{CP}$ rígido e três corpos de prova compostos ( $\mathrm{PPECC}+\mathrm{CP})$, com dimensões $355 \times 80 \times 75 \mathrm{~mm}$, conforme o método proposto por Qian e Li [8].

A partir das dimensões dos corpos de prova, utilizou-se um vão livre entre os suportes de $305 \mathrm{~mm}$ e um vão de momento constante (entre os pontos de aplicação de carga) de $100 \mathrm{~mm}$, como observado na Figura 3.

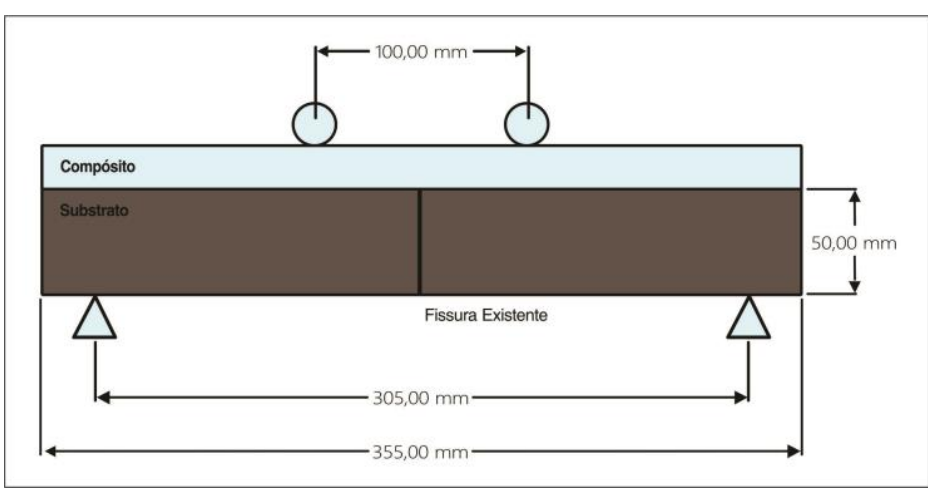

Figura 3 - Configuração do corpo de prova para análise da resistência à fissuração refletiva. 
A Figura 4 mostra o corpo de prova composto posicionado no suporte de flexão a quatro pontos.

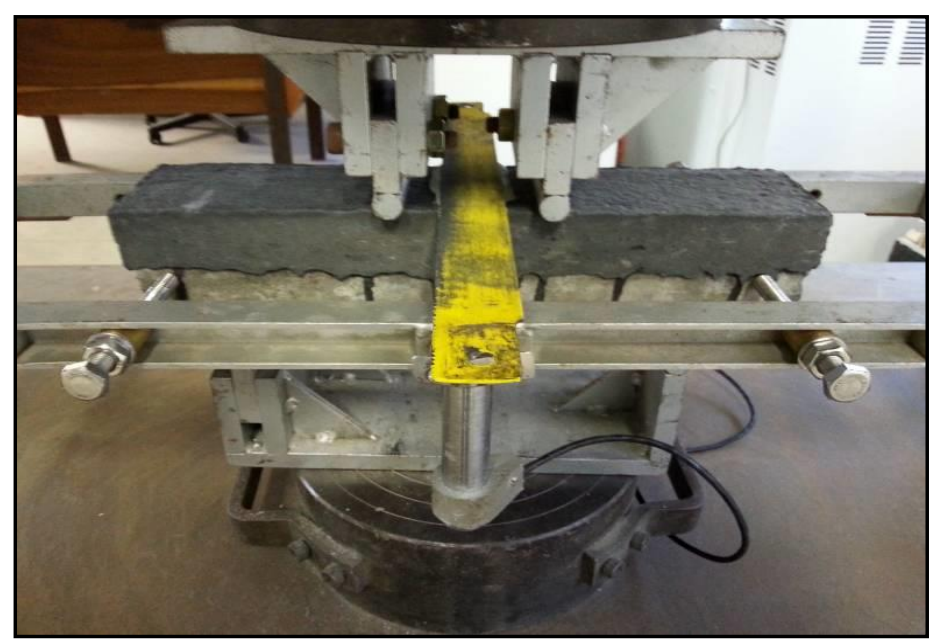

Figura 4 - Corpo de prova composto posicionado no suporte de flexão.

Nos ensaios de fadiga, foram utilizados nove corpos de prova para o traço de PPECC produzido, com dimensões idênticas aos descritos para o ensaio de flexão a quatro pontos estático, com o mesmo padrão de apoios. O carregamento caracteriza-se por um estágio de pré carregamento estático, seguido do estágio cíclico.

No primeiro estágio foi utilizado controle de deslocamentos na velocidade de $0,1 \mathrm{~mm} / \mathrm{min}$. Após este, o carregamento cíclico foi aplicado utilizando controle de carga com freqüência de $4 \mathrm{~Hz}$. Foram aplicados três níveis de carregamento em cada série de três corpos de corpos de prova do PPECC: sendo as tensões máximas iguais a $0,10,0,15$ e 0,20 do MOR do material e as tensões mínimas iguais a zero. Ou seja, a variação de tensões $(\Delta \sigma)$ em cada ciclo foi de 0 a 0,$10 ; 0$ a 0,15 ; 0 a 0,20 do MOR.

A variação de tensões e o número de ciclos resistidos pelos corpos de prova por fadiga foram utilizados para gerar a relação $\Delta \sigma-\mathrm{N}$ (variação na tensão $\mathrm{x}$ número de ciclos) dos compósitos. Foi considerado como life time máximo, ou seja, o número de ciclos para considerar a vida útil do compósito como infinita o valor de 2 milhões de ciclos [9].

A fissura produzida no substrato visa induzir a concentração de tensões na zona próxima à fissura que está em contato com a camada de overlay. Devido à presença desta falha, o substrato não contribui estruturalmente na resposta da viga composta, porém atua de forma a restringir a deformação do overlay, em função da aderência interfacial entre as camadas. experimental.

A Tabela 2 descreve a síntese do programa

Tabela 2 - Síntese do programa experimental

\begin{tabular}{c|c|c|c|c}
\hline \multirow{2}{*}{ Material } & \multicolumn{4}{c}{ Tipo de ensaio } \\
\cline { 2 - 5 } & $\begin{array}{c}\text { Estático: } \\
\text { Flexão a } 4 \\
\text { pontos }\end{array}$ & $0,10 \sigma \max$ & $0,15 \sigma \max$ & $\begin{array}{c}\text { Resistência a flexão - carregamento } \\
\text { max }\end{array}$ \\
\cline { 3 - 5 } PPECC+CP & 3 & 3 & 3 & 3 \\
CP & 3 & - & - & - \\
\hline
\end{tabular}

\section{Resultados e discussões}

\subsection{Resultados dos ensaios estáticos}

Os resultados dos ensaios de flexão a quatro pontos dos corpos de prova de CP e dos corpos de prova compostos podem ser observados nas curvas plotadas nas Figuras 5 e 6 , respectivamente. Cada curva obtida está representada por uma cor. Estes mostram que a capacidade de deflexão do PPECC estudado é superior a do CP. A alta capacidade de deflexão é consequência da adição de fibras, somada ao processo de microfissuração do compósito. Cabe ressaltar que como o Linear Variable Differential Transducers - LVDT utilizado possuía curso máximo de $5 \mathrm{~mm}$, os resultados dos gráficos são apresentados até este valor.

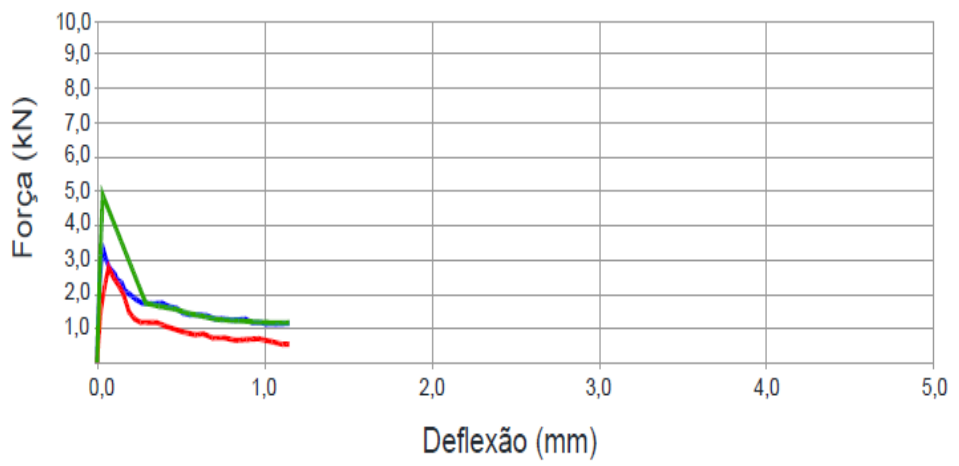

Figura 5 - Curvas tensão flexão - deflexão dos corpos de prova de concreto (CP). 


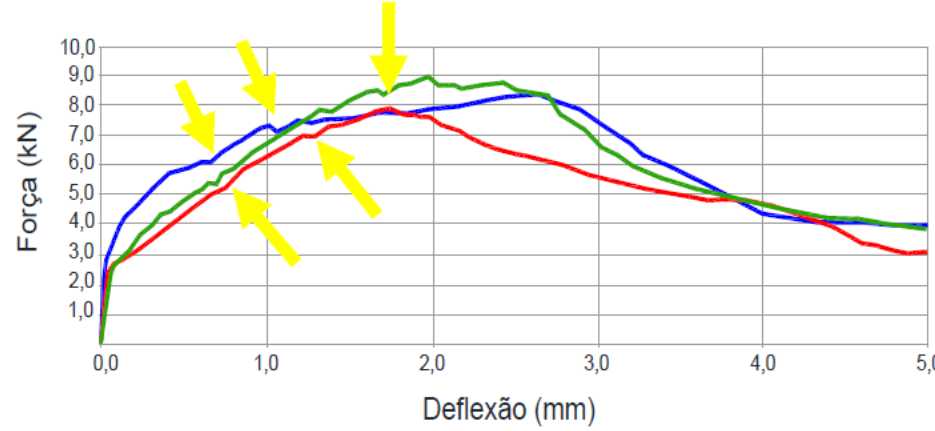

Figura 6 - Curvas tensão flexão - deflexão dos corpos de prova de concreto de PPECC.

O comportamento denominado endurecimento por deformação, ou strain-hardening, que garante a obtenção de uma resposta dúctil para o compósito, pode ser identificado na Figura 6 pelos pontos indicados pelas setas amarelas. Este comportamento é caracterizado pelas sucessivas recuperações de carga pelo compósito após a ocorrência da primeira fissura, ou o final da fase elástica. As sussessivas aberturas de fissura com consequente recuperação de carga representam a capacidade de multifissuração do compósito, que resulta na elevada capacidade de deformação.

$\mathrm{O}$ valor médio do $\mathrm{MOR}$, que representa a resistência à flexão das amostras ensaiadas pode ser observado na Tabela 3.

Tabela 3 - Valores dos módulos de ruptura.

\begin{tabular}{l|c}
\hline \multicolumn{1}{c|}{ Material } & MOR (MPa) \\
\hline Amostra de concreto (CP) & 2,38 \\
Amostra composta (PPECC $+\mathrm{CP})$ & 5,49 \\
\hline
\end{tabular}

O valor do MOR obtido para as vigas com overlay de PPECC e adição de cinza de casca de arroz estudado supera em $130,67 \%$ o valor do MOR do $\mathrm{CP}$, utilizado como referencial comparativo no ensaio de flexão. Ainda, aproxima-se do valor de MOR obtido por Garcez [3] com ECC reforçado com PP e adição de cinza volante, sendo esse de $6,18 \mathrm{MPa}$ e, praticamente, igualase ao valor de MOR obtido por Righi [10] com ECC reforçado com PP e adição de cinza de casca de arroz, sendo esse de $5,50 \mathrm{MPa}$.

A Figura 7 mostra um exemplo de configuração das fissuras para os corpos de prova compostos ensaiados à flexão.

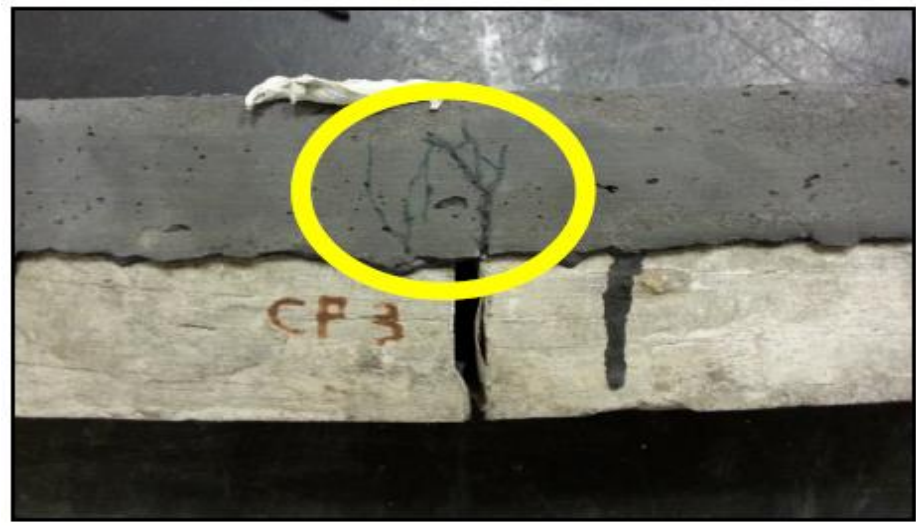

Figura 7 - Fissuração de corpo de prova composto.

Analisando a Figura 7, observam-se várias fissuras de pequena espessura, caracterizando o processo de multifissuração.

A Figura 8 mostra a fissura de um corpo de prova de $\mathrm{CP}$ ensaiado à flexão.

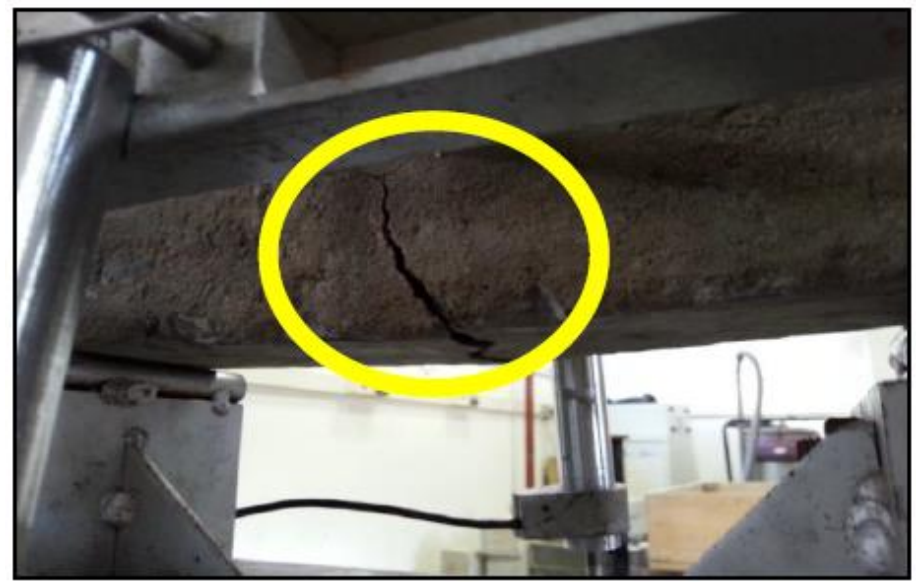

Figura 8 - Fissuração em corpo de prova de CP.

Comparando a Figura 7 e a Figura 8, pode-se notar a diferença de configuração das fissuras ocorridas nas vigas de $\mathrm{CP}$ e aquelas ocorridas no overlay de PPECC dos corpos de prova compostos. No CP ocorreu uma única fissura associada ao rompimento dos corpos de prova, enquanto que no PPECC, surgiram várias microfissuras (strain hardening), associadas a uma maior capacidade de carga e ductibilidade.

Todas as fissuras ocorrerram no terço médio dos corpos de prova ensaiados, atendendo as determinações da ASTM C7802 para consideração dos resultados obtidos. 


\subsection{Resultados dos ensaios de fadiga}

Para estudar o comportamento a fadiga do PPECC foram plotados no gráfico da Figura 9 pontos que expressam a

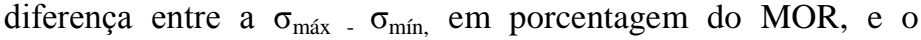
número de ciclos até a ruptura correspondentes aos resultados obtidos nos ensaios com os diferentes níveis de tensão aplicada.

Os dados da Figura 9 mostram que, como tendência, menores diferenças entre $\sigma_{\text {máx }}$ e $\sigma_{\text {mín }}$ levam a um número superior de ciclos até a falha, o que seria um resultado esperado.

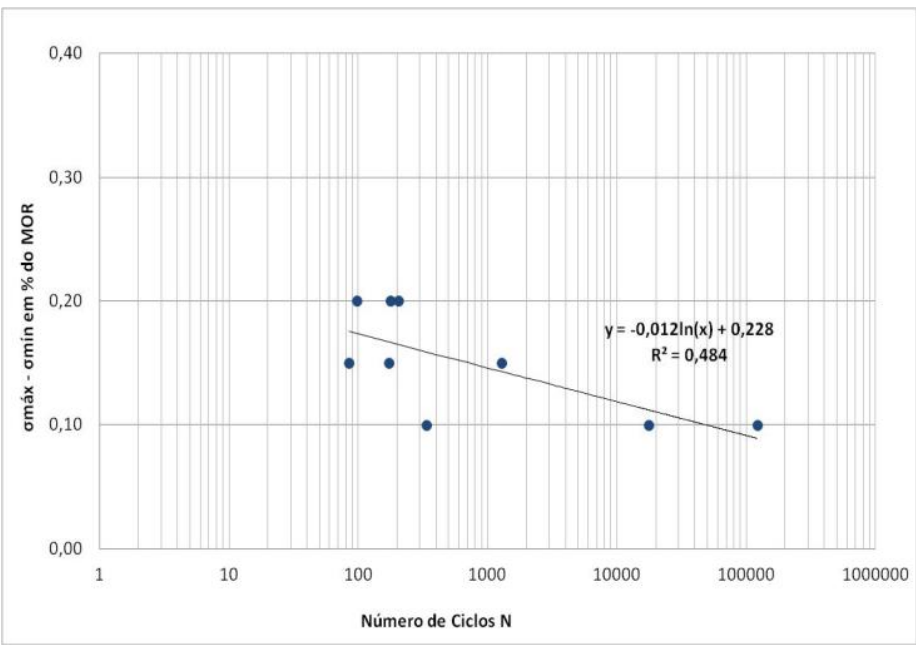

Figura 9 - Resultados dos ensaios de fadiga.

Conforme Garcez [3], a deflexão à fadiga de um material está relacionada à sua capacidade de deflexão à flexão. A evolução da deflexão depende do nível de tensão aplicado ao corpo de prova e está relacionado ao número de aberturas das fissuras que se desenvolvem no compósito. Na Figura 10 observa-se a evolução das fissuras nos corpos de prova submetidos a carregamentos de flexão estática (a) e cíclica com tensões de $10 \%$ (b), $15 \%$ (c) e $20 \%$ (d) da tensão máxima resistida pelo compósito.

Constata-se ainda que, o número de fissuras no vão central da viga (terço mediano do vão) decresce do maior nível de tensão aplicado para o menor. Comportamentos semelhantes foram registrados por Matsumoto et al. [11] e Liu, Xu e Li [12]. Esta última pesquisa registrou uma diminuição no número de fissuras na ordem de 20 vezes em compósitos de $E C C$ de elevada dureza submetidos a um nível de tensão de fadiga de 0,60 da tensão última em relação a outros submetidos a 0,80.

Os resultados desta pesquisa, corroborados pelos de pesquisas realizadas com diferentes níveis de tensão indicam que o número de fissuras desenvolvidas no overlay de ECC é inferior quando níveis mais baixos de tensão são aplicados no ensaio de carregamento cíclico, indicando que nestes casos o ECC não desenvolve o comportamento strain hardening com consequente múltipla fissuração.

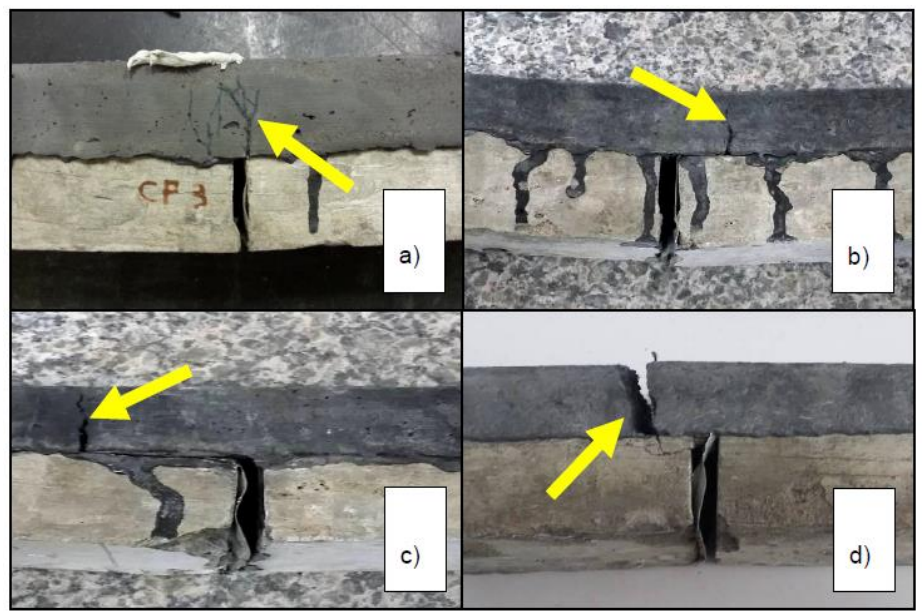

Figura 10 - Fissuração dos corpos de prova: (a) ensaio estático; (b) com $\sigma_{\max } 10 \%$ do MOR; (c) com $\sigma_{\max } 15 \%$ do MOR; (d) com $\sigma_{\max } 20 \%$ do MOR.

Na Figura 11 constam dados de ensaios de fadiga em ECC realizados por diferentes pesquisadores [8-9, 13-15], incluindo os produzidos nesta pesquisa. Os ensaios de fadiga são influenciados por muitos fatores, dos quais se pode destacar a frequência aplicada nos ciclos de carga, os tipos de materiais presentes nos compósitos, os modos de carregamento, a variação entre $\sigma_{\text {máx }}$ e $\sigma_{\text {mín }}$, entre outros. Desta forma, os dados gerados possuem grande dispersão, o que torna difícil a construção e análise de um banco de dados de fadiga.

A Figura 11 mostra claramente que há uma tendência de obtenção de melhores resultados (maior número de ciclos até a ruptura) para variações entre $\sigma_{\text {máx }}$ e $\sigma_{\text {mín }}$ em torno de 40 e $70 \%$ do MOR. Verifica-se que quando as tensões aplicadas são muito baixas, como as utilizadas no presente trabalho, com o ECC ainda atuando na fase elástica, o estágio de múltipla fissuração do material não é alcançado. Por outro lado, tensões de fadiga muito elevadas, quando o processo de microfissuração está muito adiantado, também tendem a ocasionar ruptura do material com um número baixo de ciclos.

$\mathrm{Na}$ figura 12, o mesmo banco de dados de ensaios de fadiga realizados em $E C C$ da figura 11 foi utilizado, destacando ensaios realizados em PPECC (círculos em vermelho) em PVAECC (triângulos em preto). 

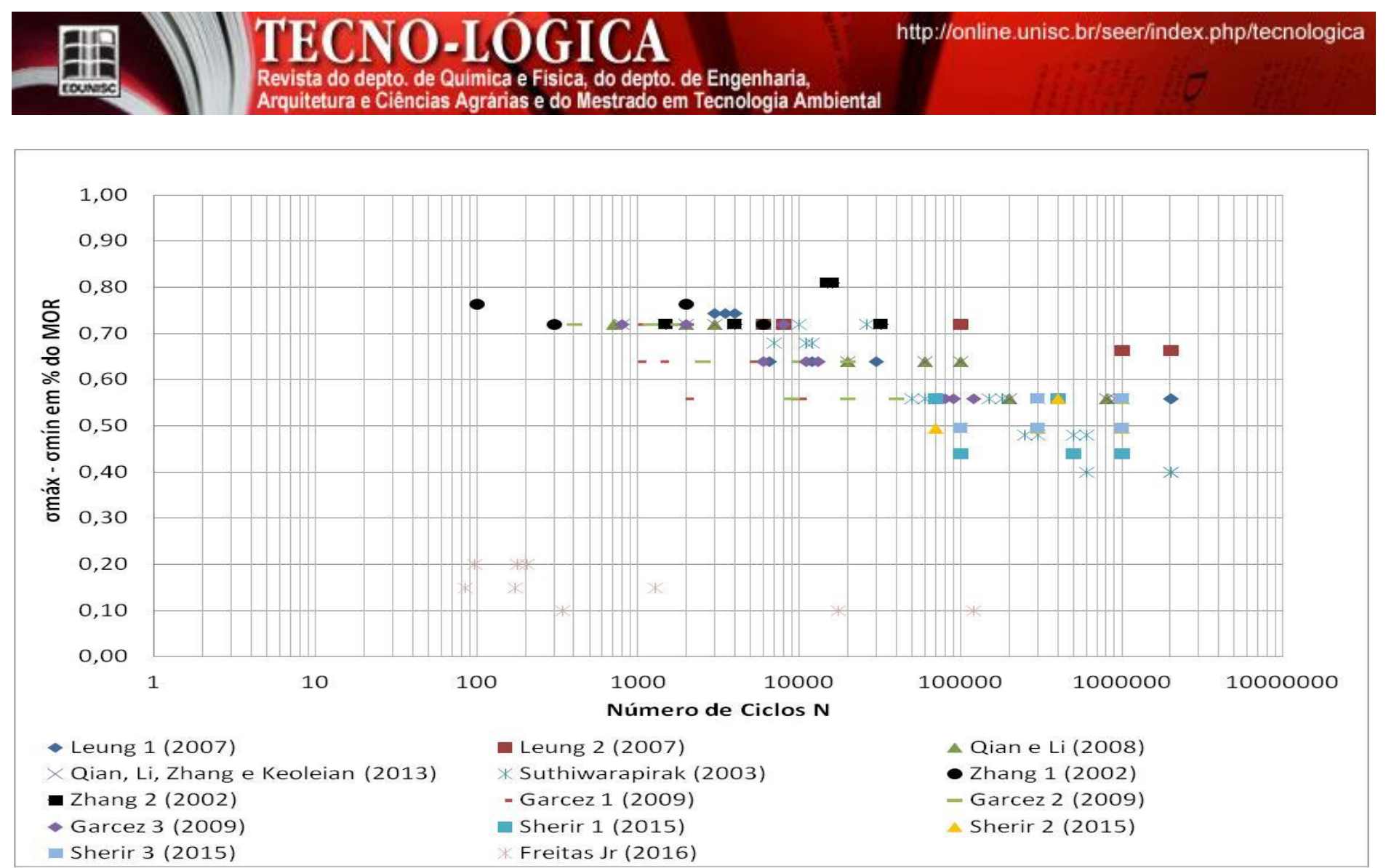

Figura 11 - Demonstração gráfica de resultados de ensaios de fadiga realizados

em ECC de diversos trabalhos sobre o assunto

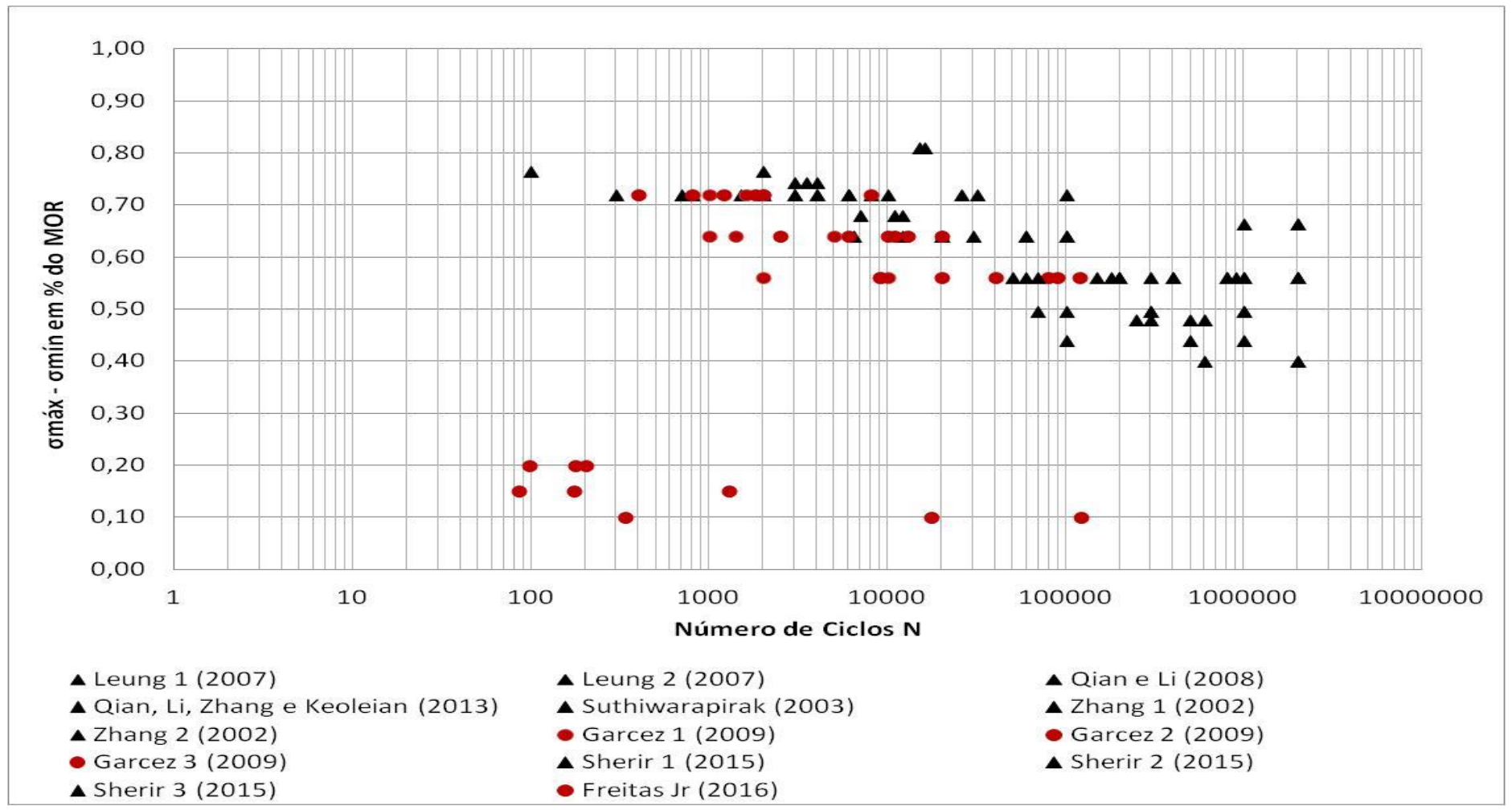

Figura 12 - Demonstração gráfica de resultados de fadiga em ECC destacando

ensaios realizados em PPECC e PVAECC. 
O baixo custo quando comparadas com as fibras de PVA, a ductilidade, a superfície hidrofóbica e a facilidade de dispersão são características que tornam as fibras de PP adequadas para incorporação em um compósito cimentício, segundo Raivio e Sarvantra [16] e Zhang et al. [17].Por outro lado, a natureza apolar das fibras de PP pode prejudicar a aderência do compósito [18], uma vez que a pasta de cimento apresenta natureza polar. Resultados de Pakravan et al. [19] mostraram que apesar de haver um intertravamento entre as fibras de PP e a matriz de cimento, existe deficiência da adesão química entre as fibras de PP e os produtos de hidratação do cimento. Entretanto, os efeitos das interações mecânicas na energia de adesão entre as fibras de PP e a matriz cimentícia se mostraram cerca de 100 vezes superiores aos efeitos das interações químicas, o que indica que neste caso a ligação mecânica entre as fibras e a matriz exerce um papel mais importante que a aderência química.

Os dados da figura 12 mostram que em geral os PPECC apresentam comportamento inferior à fadiga, quando comparados aos PVAECC.

\section{Conclusões}

Considerando os dados experimentais e as constatações descritas acima, pode-se chegar afirmar que é viável a produção de compósitos do tipo ECC com fibras de PP fabricadas e disponíveis no Brasil, e cuja composição da matriz cimentícia tenha a incorporação de cinza de casca de arroz.

O PPECC com adição de cinza de casca de arroz como material suplementar apresentou desempenho satisfatório aos ensaios de flexão estática, tendo uma capacidade de deflexão 2,3 vezes maior que o $\mathrm{CP}$, sendo esta a característica desejada no comportamento à flexão deste compósito. Ainda, foi possível identificar o efeito strain-hardening nos corpos de prova de PPECC, a partir das quedas de carga ao longo das curvas de flexão.

Quanto ao comportamento nos ensaios de flexão cíclica, dado o nível de tensão aplicado nos ensaios realizados, o ECC não atingiu os resultados esperados. Quanto menor o nível de tensão aplicada, menor será o número de fissuras desenvolvidas no overlay de PPECC e este não desenvolve suas potencialidades, tais como, o efeito de microfissuração característico desse tipo de compósito e sua ductibilidade. Entretanto, foi possível analisar através dos mesmos, o comportamento do material na sua fase elástica.

Os resultados deste trabalho, em conjunto com o banco de dados de outros trabalhos já realizados, permite concluir que o ECC constitui-se em um compósito alternativo com viabilidade de aplicação em overlays sobre base de CP sujeitos a esforços cíclicos.

\section{EVALUATION OF THE FATIGUE RESISTANCE OF ENGINEERED CEMENTITIOUS COMPOSITES (ECC), REINFORCED WITH POLYPROPYLENE FIBER AND PRODUCED WITH ADDITION OF RICE BARK ASH.}

ABSTRACT: The Engineered Cementitious Composites (ECC) is a special type of cementitious composite of high-performance reinforced with fibre, whose main characteristic is the high ductility. The ECC feature is composed of cement, pozzolanic material, fine aggregate and fibres. This study aimed to evaluate the fatigue resistance of Engineered Cementitious Composites traits reinforced with polypropylene fibres - PPECC - and which composition of the cementitious matrix has the incorporation of rice hull ash as supplementary material. For this, test specimens with concrete substrate for pavement and overlay were produced with the PPECC trait developed. The specimens were submitted to the four points (static and cyclic). The results were be compared with the static test PPECC traits with the results of this same test with test specimens produced only by concrete pavers. The PPECC of this study presented satisfactory performance in the static flexion tests. The results of cyclic testing at different intervals stress were analysed. The fatigue behavior of the composite was evaluated with the results of the stress variation $\mathrm{x}$ cycle numbers obtained in the cyclic test.. In the cyclic flexural tests, it can be observed that the lower the applied stress level, the smaller the number of cracks developed in the PPECC overlay and the latter does not develop its potentialities. As a conclusion, we have that the ECC is an alternative composite with viability of application in overlays on the base of $\mathrm{CP}$ subjected to cyclic efforts.

Key-words: Cementitious Composites; pozzolanic materials; composite cement with the addition of polypropylene fibres.

\section{Referências}

[1] QIAN, S.; LI, V. C. Durable pavement with ECC. In: INTERNATIONAL CONFERENCE ON MICROSTRUCTURE RELATED DURABILITY OF CEMENTITIOUS COMPOSITES, 1, Nanjing, China. Anais...Nanjing, China: 2008. p.535-543.

[2] CASAGRANDE, E. Q. Compósito reforçados com fibras de polipropileno e com adição de cinza volante: estudo do desempenho em pavimento de concreto. 2012. 78f. Dissertação (Programa de Pós-graduação e Engenharia Civil Mestrado) - Universidade Federal do Rio Grande do Sul, Porto Alegre. 2012.

[3] GARCEZ, E. O. Investigação do Comportamento de Engineered Cementitious Composites Reforçados com Fibras de Polipropileno como Material para Recapeamento de Pavimentos. 2009. 176f. Tese (Programa de Pós-Graduação em Engenharia Civil - Doutorado) - Universidade Federal do Rio Grande do Sul, Porto Alegre. 2009

[4] KAMADA, T.; LI, V. C. The Effects of Surface Preparation on the Fracture Behavior of ECC/Concrete Repair System, Journal of Cement and Concrete Composites, Vol. 22, n. 6, p.423-431, 2000. 
[5] DOWLING, N. E. Mean Stress Effects in Stress-Life and Strain-Life Fatigue. Blacksburg, Virginia, USA: Department of Engineering Science and Mechanics Virginia Polytechnic Institute and State University, 2002.

[6] ASSOCIAÇÃO BRASILEIRA DE NORMAS TÉCNICAS. NBR NM 67 Concreto - Determinação da consistência pelo abatimento do tronco de cone. Rio de Janeiro: ABNT, 1998.

[7] AMERICAN SOCIETY FOR TESTING AND MATERIALS. ASTM C78 02: Standard test method for flexural strength of concrete. West Conshohocken, Pennsylvania: ASTM, 2002

[8] QIAN, S.; LI, V. C.; ZHANG, H.; KEOLEIAN, G. A. Life cycle analysis of pavement overlays made with Engineered Cementitious Composites. Cement \& Concrete Composites Journal, Vol.35, p. 78-88, 2013.

[9] LEUNG, C. K. Y.; CHEUNG, Y. N.; ZHANG, J. Fatigue enhancement of concrete beam with ECC layer. Cement and Concrete Research, Vol.37, p.743$750,2007$.

[10] RIGHI, Débora Pedroso. Caracterização do Comportamento Mecânico de Compósitos Cimentícios de Elevada Deformação com a Utilização de Fibras de Polipropileno e Cinza de Casca de Arroz. 2015. 176f. Dissertação (Programa de Pós-Graduação em Engenharia Civil - Mestrado) - Universidade Federal do Rio Grande do Sul, Porto Alegre, 2015.

[11] MATSUMOTO, T.; SUTHIWARAPIRAK, P.; KANDA, T. Mechanisms of Multiple Cracking and Fracture of DFRCCs under Fatigue Flexure. In: PROCEEDINGS OF THE JCI INTERNATIONAL WORKSHOP ON DUCTILE FIBER REINFORCED CEMENTITIOUS COMPOSITES (DFRCC) APPLICATION AND EVALUATION, Takayama, Japan, p. 259-268, 2002.

[12] LIU, W.; XU, S.; LI, H. Flexural fatigue damage model of ultra-high toughness cementitious composites on base of continuum damage mechanics. International Journal of Damage Mechanics, Vol.23, n .7, p.949-963, 2014.

[13] SHERIR, M. A. A.; HOSSAIN, K. M. A.; LACHEMI, M. Structural Performance of Polymer Fiber Reinforced Engineered Cementitious Composites Subjected to Static and Fatigue Flexural Loading. Polymers, Vol.7, p.1299-1330, 2015.

[14] ZHANG, J.; LI, V. C. Monotonic and fatigue performance in bending of fiber-reinforced engineered cementitious composite in overlay system. Cement and Concrete Research, Vol.32, p.415-423, 2002.

[15] SUTHIWARAPIRAK, P.; MATSUMOTO, T.; KANDA, T. Flexural fatigue failure characteristics of an engineered cementitious composite and polymer cement mortars. University of Tokyo. Japan, 2003.

[16] RAIVIO, P; SARVARANTA, L. Microstructure of fibre mortar composites under fire impact - effect of polypropylene and polyacrylonitrile fibres, Cement and Concrete Research, Vol. 24, n ${ }^{\circ}$ 5, pp. 896-906, 1994.

[17] ZHANG, R. et al. Application of PP-ECC in beam-column joint connections of rigid-framed railway bridges to reduce transverse reinforcements, Engineering Structures, Vol. 86, pp.146-156, 2015.

[18] BUENDÍA, A. M. L. et al. Surface treated polypropylene (PP) fibres for reinforced concrete. Cement and Concrete Research, Vol.54, p.29-35, 2013.

[19] PAKRAVAN, H. R.; JAMSHIDI, M.; LATIFI, M. Adhesion of Polypropylene Fiber to Cement Matrix. J. Adhesion Sci. Technol., Vol. 1, p.1-11, 2012. 